\title{
An Efficient Content Based Image Retrieval System
}

\author{
Dr. Meenakshi Sharma ${ }^{1}$, Anjali Batra ${ }^{2}$ \\ ${ }^{I}$ (Department of Computer Science and Applications, HCTM, Kaithal, Haryana, India) \\ ${ }^{2}$ (Department of Computer Sc. and Engineering, HCTM, Kaithal, Haryana, India)
}

\begin{abstract}
Content based image retrieval is an active research issue that had been famous from 1990s till present. The main target of CBIR is to get accurate results with lower computational time. This paper discusses on the comparative method used in color histogram based on two major methods used frequently in CBIR which are; normal color histogram using GLCM, and color histogram using KMeans. A set of 9960 images are used to test the accuracy and the precision of each methods. Using Euclidean distance, similarity between queried image and the candidate images are calculated. Experiment results shows that color histogram with K-Means method had high accuracy and precise compared to GLCM. Future work will be made to add more features that are famous in CBIR which are texture, color, and shape features in order to get better results.
\end{abstract}

Keywords: CBIR, Euclidean distance, GLCM, KMeans, Quadratic distance.

\section{Introduction}

Content-based image retrieval, a technique which uses visual contents to search images from image banks scale according to the interests of users, has been an area of active research and rapid progress since the early 1990. During the last decade, have made remarkable progress in theoretical research and system development. However, there are still many difficult research problems continue to attract researchers from multiple disciplines.

Before the introduction of the basic theory of recovery based on the content, we 'll take a brief look at its development. The first work on image retrieval can be traced back to the late 1970s. In 1979, a conference on technical database for pictorial applications took place in Florence. Since then, the potential application of image database [1] management techniques has attracted the attention of researchers. Early techniques based on visual characteristics generally not been but on textual annotation of images. In other words, the images were first annotated with text and then searched using a text-based approach of traditional management systems database. Comprehensive studies of the first methods of image retrieval based text can be found. Text-based image retrieval techniques use traditional databases to manage images. With text descriptions, images can be organized by topical or semantic hierarchies to facilitate easy navigation and based on standard Boolean queries navigation. However, when the automatic generation of descriptive texts for a wide range of images is not possible, the most image -based text retrieval systems require manual annotation of images. Obviously, manual annotation of images is a time consuming and expensive for large image databases task, and is often subjective, incomplete and context. Consequently, it is difficult for traditional methods based on a text to support a variety of applications dependent tasks. In the early 1990s, due to the advances of the Internet and new technologies of digital image sensors, the volume of digital images produced by medical, industrial, and other scientific applications, education, available for users has increased dramatically. The difficulties encountered by the textbased retrieval is becoming more severe. Effective management of rapidly expanding visual information became an urgent problem. The need for training of the driving force behind the emergence of [2] based on the contents of the recovery techniques of the image. In 1992, the National Science Foundation of the United States organized a workshop on the management systems of visual information identification to identify new directions in management systems based on image data. It was widely recognized that more efficiently and intuitively to represent and index visual information are based on the properties that are inherent in the images themselves. Researchers communities of computer vision, management database, the man -machine interface, and information retrieval have been attracted to this area. Since then, research on image retrieval recovery grew rapidly. Since 1997, the number of research publications on techniques for extracting visual information of the organization [3], indexing, search, and user interaction, and database management data has increased enormously. Similarly, a large number of systems of academic and commercial research have been developed by universities, government agencies, businesses and hospitals. Comprehensive studies on these techniques and systems can be found. Images by the contents of recovery, using the visual content of an image: as color, shape, texture and spatial layout to represent and index the image. In recovery systems typical content -based image, the visual content of the images in the database are extracted and described by multi-dimensional feature vectors The feature vectors of the images in the database constitute a database characteristic. To retrieve images, users provide the system an example recovery [4] outlines images or figures. The system then passes these examples in its internal representation of feature vectors. The similarities / distances between the feature vectors of the 
query example or sketch and those images in the database [5] are then calculated and retrieval is performed using an indexing system [6]. The indexing scheme provides an efficient [7] means for searching the image database. Recent research systems have integrated the relevance feedback from users to modify the recovery process to generate semantically perception and the results of the most significant recovery.

\section{Proposed Design Analysis}

The search begins with providing an image as the 'query image' [8]. The objective is to retrieve images which are similar to the query image. The features that were considered initially include, color, texture and entropy. Different implementations of CBIR make use of different types of user queries. Query by example [9] is a query technique that involves providing the CBIR system with an example image that it will then base its [10] search upon. The underlying search algorithms may vary depending on the application, but result images should all share common elements with the provided example. Options for providing example images to the system include: A pre-existing image may be supplied by the user or chosen from a random set. The user draws a rough approximation of the image they are looking for, for example with blobs of color or general shapes. This query technique removes the difficulties that can arise when trying to describe images with words. The CBIR technique called semantic retrieval starts with a user making a request like "find pictures of Abraham Lincoln". This type of open-ended task is very difficult for computers to perform- Lincoln may not always be facing the camera or in the same pose. Many CBIR systems therefore generally make use of lower-level features like texture, color, and shape. These features are either used in combination with interfaces that allow easier input of the criteria or with databases [11] that have already been trained to match features [12] (such as faces, fingerprints, or shape matching). However, in general, image retrieval requires human feedback in order to identify higher-level concepts. Combining CBIR search techniques available with the wide-range of potential users and their intent can be a difficult task. An aspect of making CBIR successful relies entirely on the ability to understand the user intent. CBIR systems can make use of relevance feedback [13], where the user progressively refines the search results by marking images in the results as "relevant", "not relevant", or "neutral" to the search query, then repeating the search with the new information. The most common method for comparing two images in content-based image retrieval (typically an example image and an image from the database) is using an image distance measure. An image distance measure compares the similarity of two images in various dimensions such as color, texture, shape, and others. For example a distance of 0 signifies an exact match with the query, with respect to the dimensions that were considered. As one may intuitively gather, a value greater than 0 indicates various degrees of similarities between the images. Search results then can be sorted based on their distance to the queried image. Many measures of image distance (Similarity Models) have been developed. Computing distance measures based on color similarity is achieved by computing a color histogram for each image that identifies the proportion of pixels within an image holding specific values. Examining images based on the colors they contain is one of the most widely used techniques because it can be completed without regard to image size or orientation. However, research has also attempted to segment color proportion by region and by spatial relationship among several color regions. The search begins with providing an image as the 'query image' [14]. The objective is to retrieve images which are similar to the query image. The features that were considered initially include, color, texture and entropy. One of the most important features that make possible the recognition [15] of images by humans is color. Color is a property that depends on the reflection of light to the eye and the processing of that information in the brain. We use color every day to tell the difference between objects, places, and the time of day. Usually colors are defined in three dimensional color spaces. These could either be RGB (Red, Green, and Blue), HSV (Hue, Saturation, and Value) or HSB (Hue, Saturation, and Brightness). Color combination can be easily visualized using histograms. A color histogram is a type of bar graph, where each bar represents a particular color of the color space being used. Texture measures look for visual patterns in images and how they are spatially defined. Textures are represented by texels which are then placed into a number of sets, depending on how many textures are detected in the image. These sets not only define the texture, but also where in the image the texture is located. Texture is a difficult concept to represent. The identification of specific textures in an image is achieved primarily by modeling texture as a two-dimensional gray level variation. The relative brightness of pairs of pixels is computed such that degree of contrast, regularity, coarseness and directionality may be estimated. The problem is in identifying patterns of co-pixel variation and associating them with particular classes [16] of textures such as silky, or rough. Fig. 1 shows the basic design of our CBIR system. We retrieve images in three stages, in the initial stage, we retrieved images with respect to the color, if the user is satisfied with the retrieval results, we stopped there itself, thereby not giving much work to the system. If not, the retrieval was carried out based on the texture of the images (we calculated the overall pixel intensity of the images.). If the user is still not satisfied with the results, we calculated the entropy of images before comparing them. 


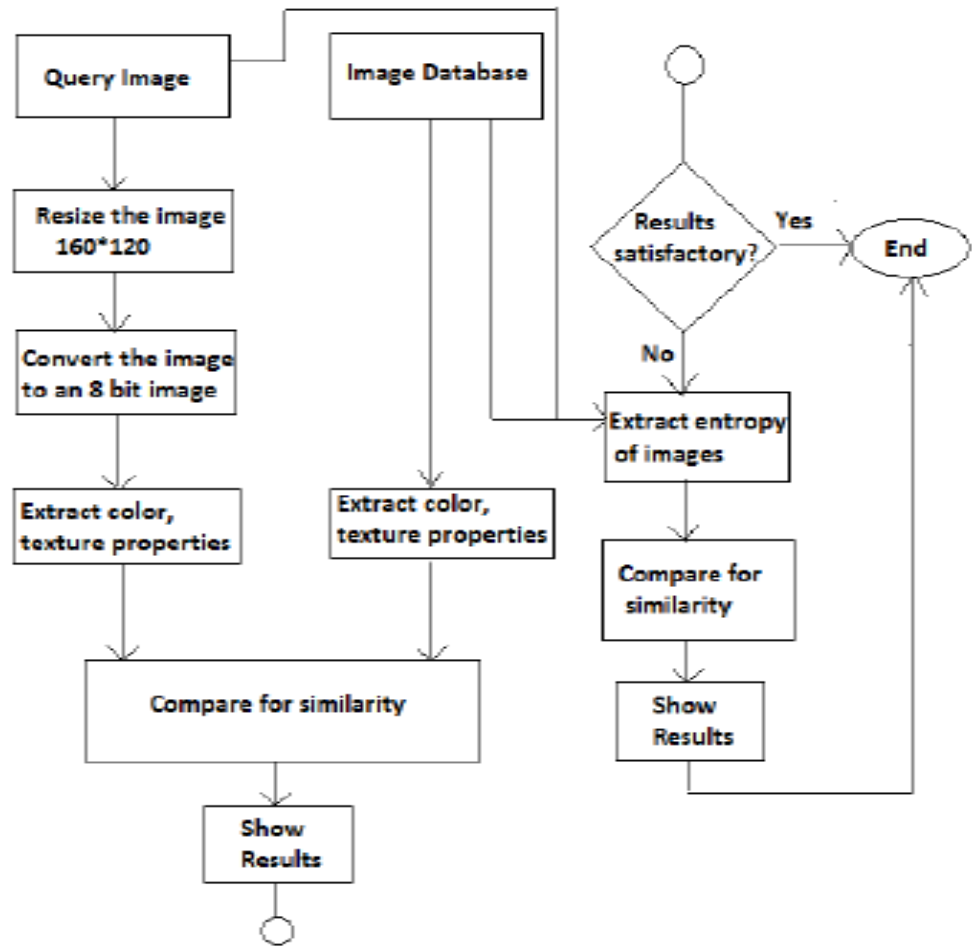

Figure1. Design Diagram

The bars in a color histogram are referred to as bins and they represent the $\mathrm{x}$-axis. The number of bins depends on the number of colors that are in an image. The $y$-axis denotes the number of pixels there are in each bin. In other words how many pixels in an image are of a particular color. A sample histogram for an image has been shown in the Fig. 2.
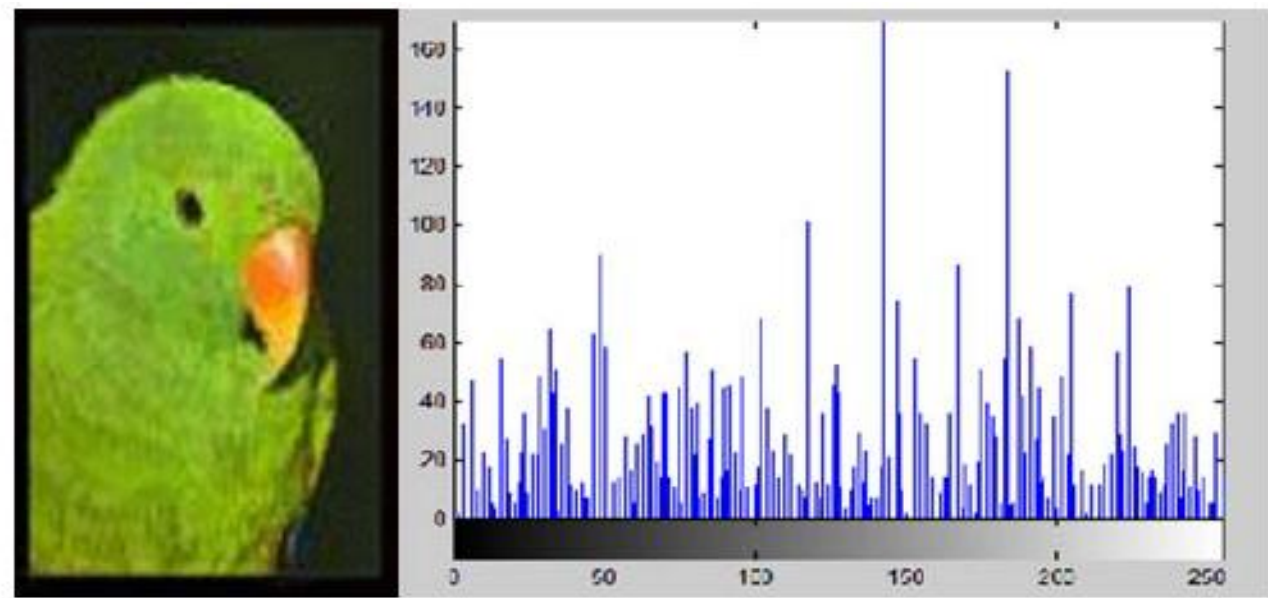

Figure 2. Sample image and its histogram

An image texture is a set of metrics calculated in image processing designed to quantify the perceived texture of an image. Image Texture gives us information about the spatial arrangement of color or intensities in an image or selected region of an image. Image textures can be artificially created or found in natural scenes captured in an image. Image textures are one way that can be used to help in Segmentation (image processing) or classification of images. To analyze an image texture in computer graphics, there are two ways to approach the issue: Structured Approach and Statistical Approach. A structured approach sees an image texture as a set of primitive texels in some regular or repeated pattern. This works well when analyzing artificial textures. To obtain a structured description a characterization of the spatial relationship of the texels is gathered by using Voronoi tessellation of the texels. A statistical approach sees an image texture as a quantitative measure of the arrangement of intensities in a region. In general this approach is easier to compute and is more widely used, since natural textures are made of patterns of irregular sub elements. The use of edge detection to 
determine the number of edge pixels in a specified region helps determine a characteristic of texture complexity. After edges have been found the direction of the edges can also be applied as a characteristic of texture and can be useful in determining patterns in the texture. These directions can be represented as an average or in a histogram. Consider a region with $\mathrm{N}$ pixels. the gradient-based edge detector is applied to this region by producing two outputs for each pixel p: the gradient magnitude $\operatorname{Mag}(\mathrm{p})$ and the gradient direction $\operatorname{Dir}(\mathrm{p})$. The edgeness per unit area can be defined by $F_{\text {edgeness }}=\frac{|\{p \mid \operatorname{Mag}(p)>T\}|}{N}$ To include orientation with edgeness we can use histograms for both oradient magnitude and gradient direction. Let $\mathrm{H}_{\mathrm{mag}}(\mathrm{R})$ denote the normalized histogram of gradient magnitudes of region $\mathrm{R}$, and let $\mathrm{H}_{\mathrm{dir}}$ denote the normalized histogram of gradient orientations of region $\mathrm{R}$. Both are normalized according to the size ${ }_{\mathrm{N}_{\mathrm{R}} \text { Then }} F_{\text {magdir }}=\left(H_{\text {mag }}(R), H_{\text {dì }}(R)\right)_{\text {is }}$ quantitative texture description of region R. Cooccurrence matrix captures numerical features of a texture using spatial relations of similar gray tones. Numerical features computed from the co-occurrence matrix can be used to represent, compare, and classify textures. The following are a subset of standard features derivable from a normalized co-occurrence matrix:

$$
\begin{aligned}
\text { Angular 2nd Moment } & =\sum_{i} \sum_{j} p[i, j]^{2} \\
\text { Contrast } & =\sum_{n=0}^{N g-1} n^{2}\left\{\sum_{i=1}^{N g} \sum_{j=1}^{N g} p[i, j]\right\}, \text { where }|i-j|=n \\
\text { Correlation } & =\frac{\sum_{i=1}^{N g} \sum_{j=1}^{N g}(i j) p[i, j]-\mu_{x} \mu_{y}}{\sigma_{x} \sigma_{y}} \\
\text { Entropy } & =-\sum_{i} \sum_{j} p[i, j] \log (p[i, j])
\end{aligned}
$$

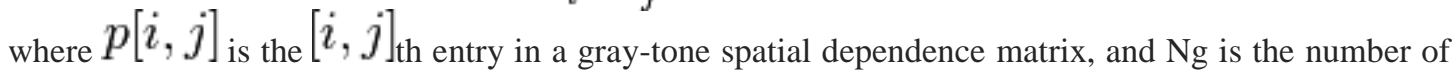
distinct gray-levels in the quantized image. One negative aspect of the co-occurrence matrix is that the extracted features do not necessarily correspond to visual perception. Comparing images based on color alone will not be sufficient for efficient retrieval. Hence, another property called, 'texture' is taken into account. Texture describes the physical composition of the picture. We have made use of the wavelet transform technique to analyze the texture of the image. Wavelet [17] is a small wave and wavelet transformation is the process of converting a signal into a series of wavelets. This technique was very helpful to obtain coarser information from the image that is not readily available in the raw image and helped in analyzing the image and identifying patterns on it. The main objective of the wavelet [18] transformation was to calculate the pixel intensity of the images, based on which, the further comparisons can be made. Since MatLab has built-in functions for wavelet transformation; it was very easier for us to carry out our work. We first decomposed the image into 4 sub-bands (low-low, low-high, high-low, high-high bands) each of different frequency. We observed that, the frequency was concentrated much in the low-low sub band and used the same for further decompositions. We considered almost all kinds of wavelet methods available for decomposition [19]. Of those, we found that, Daubechies wavelet proved to provide us good results. In particular, we incorporated ' $d b 10$ ' for our testing purposes. The following Fig. 3 shows the wavelet function as a graph.

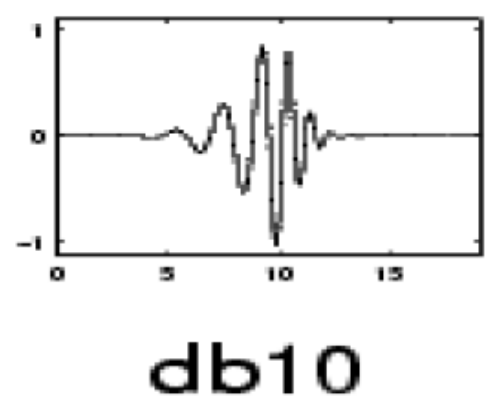

Figure 3. Daubechies wavelet 
Similarity is a measure of distance between image features. Fig. 4 shows the similarity of a query image to two images. Different similarity measures are described in literature, the commonly used in CBIR systems are Minkowski distance, Euclidean distance and the quadratic distance metric. The commonly used method of evaluating the performance of a CBIR system is Precision and Recall Graphs. The precision and recall are the measures of the effectiveness and robustness of the system.

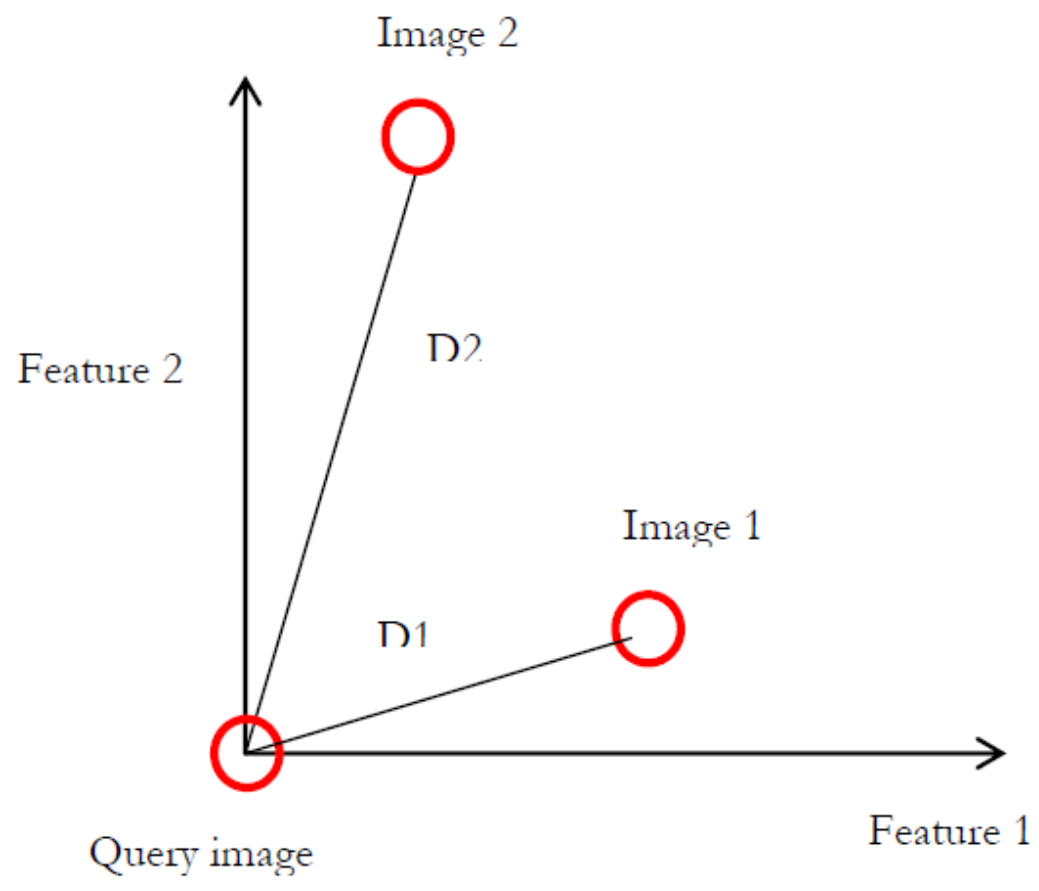

Figure 4. Similarity distance

\section{Image Retrieval Through Relevance Feedback}

Most of the currently available CBIR systems require the end user to provide feedback to the application, in the sense, the end user is expected to train the CBIR system with some positive images which are visually similar to the query image and, some negative images which are in no way related to the query image. This approach, though seems to be beneficial for the application to study the query image in detail, it consumes much of the user's time in making them interact with the application in a long duration. This will ultimately make the end user tired of following the process, which is not the expected response. Hence, we have excluded the relevance feedback [20] option, and introduced the entropy based retrieval.

\section{Image Conversion}

Before uploading an image into the image database, we had to check its specifications. If the image doesn't correspond to our requirement of 256 bit, we had to convert it into an image of bit depth equal to 8 . We used rgb2ind () method for achieving this. One more important prerequisite to deal with images in MatLab is the image size. We chose the dimension $160^{*} 120$. We used the built-in function imresize () to automatically convert the images before uploading into the database.

\section{Performance And Results}

The database had nearly 500 images all of type 256 bit '.bmp's, and size $160 * 120$; Fig. 5 shows a portion of database. 


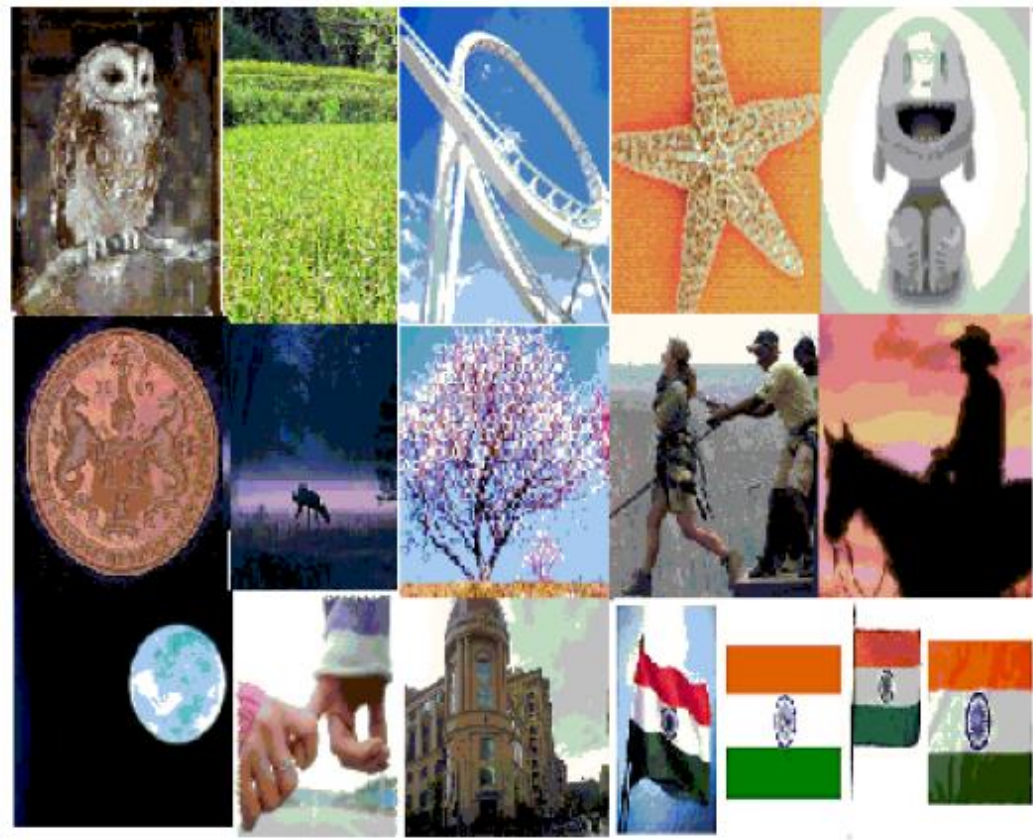

Figure 5. Images in the database

Fig. 6 shows the query image as follows.

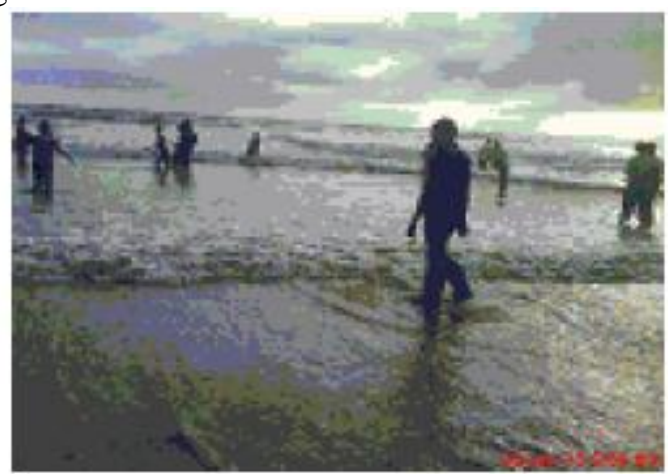

Figure 6. Query Image

The above image is processed with respect to color. Following steps hold good. Histogram [4] will be generated first using imhist () method. Fig. 7 shows the snapshot of the same. The above step is repeated for all the images in the database images. Quadratic distance is found between each pair of images. Distance value is sorted. Top most results are displayed.

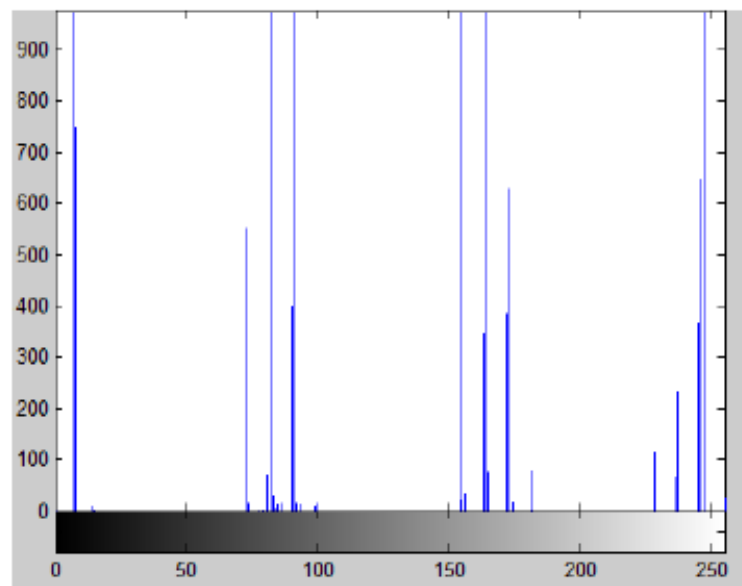

Figure 7. Histogram generated for Fig. 6 


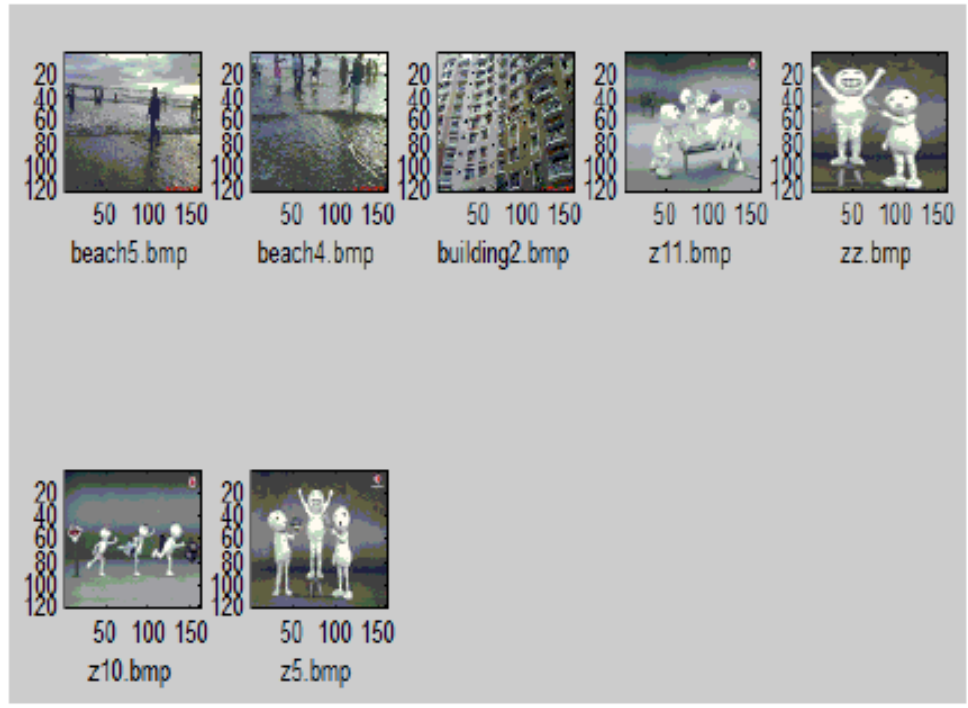

Figure 8. Sample output for color search

Following graph contained in Fig. 9 shows the quadratic distance between images. In the texture portion of the work, the query image is decomposed into 4 sub bands for 6 times (6 levels of decomposition for getting coarser information). So also the images in the database are decomposed. Fig. 10 shows a sample decomposed image.

Finally, the energy (the pixel intensity) of each decomposed image is calculated. A graph showing the Euclidean distance between the images is shown in Fig. 11. Final step is to find the Euclidean distance between query image and every image in the database. Fig. 12 shows the sample output. If the user is not satisfied with the results, he/she can opt for entropy based image retrieval. There, only exactly matching images will be displayed. Fig. 13 shows the entropy based image retrieval. As it can be seen from the execution results, color based retrieval focuses only on color factor. The efficiency is less than 50\%, whereas in texture [21] based image retrieval, it was improved to $75 \%$. Finally, in entropy based image retrieval, the efficiency is nearly $95 \%$.

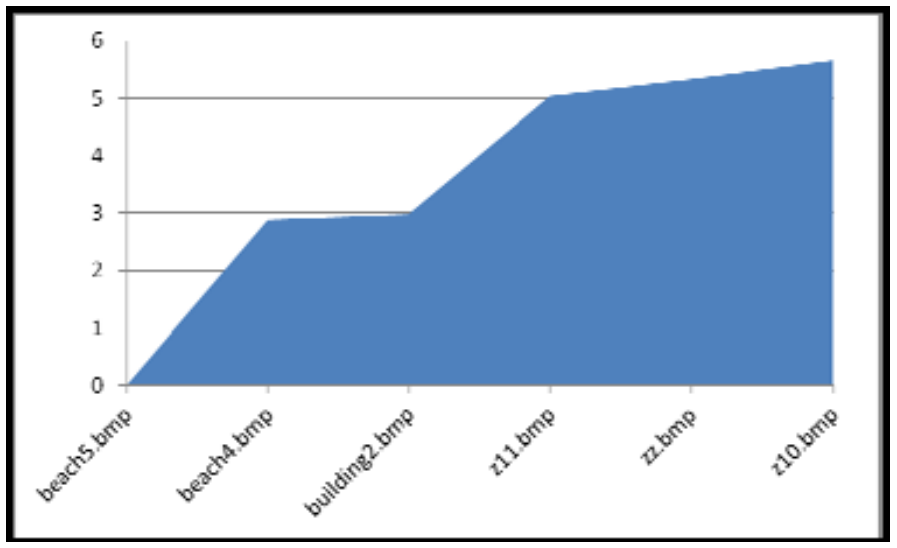

Figure 9. Graph of quadratic distance between images.

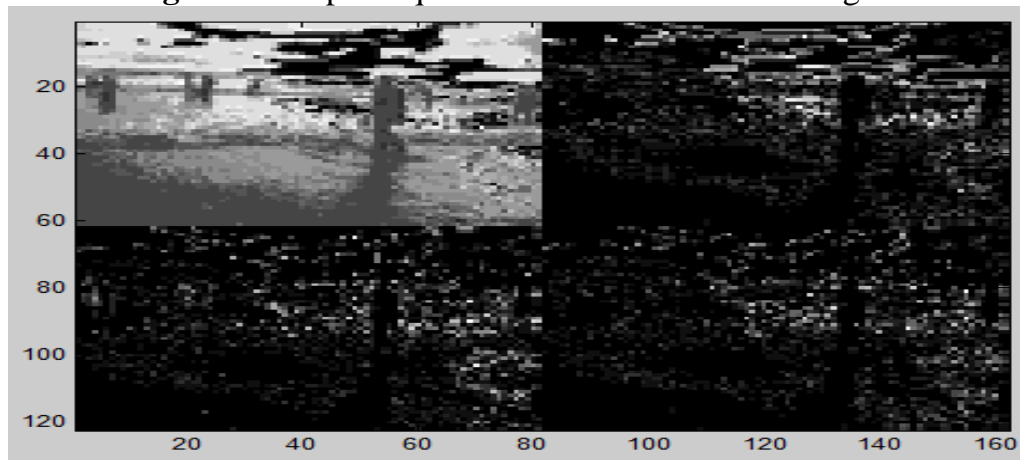

Figure 10. Decomposed image 


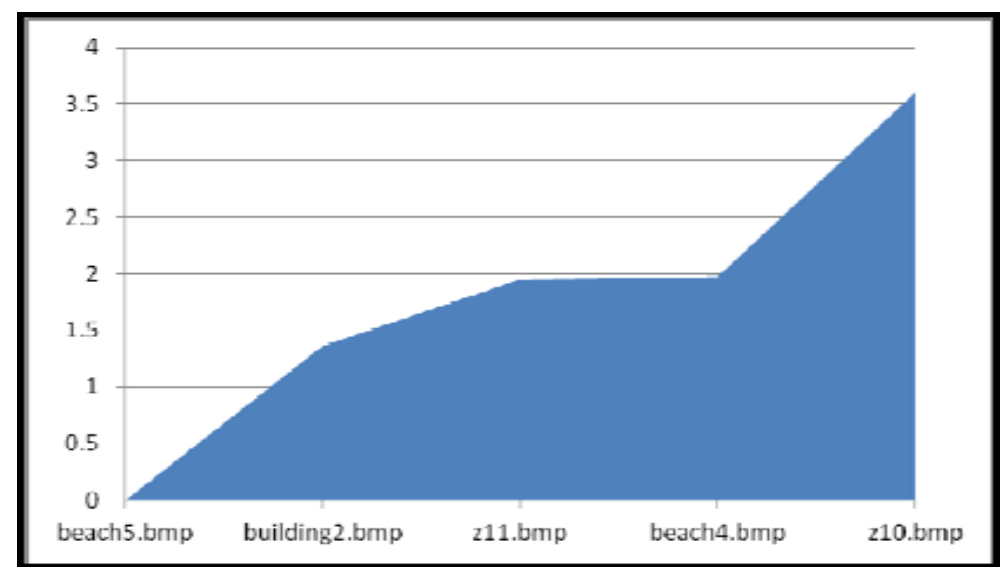

Figure 11. Euclidean distance between image.
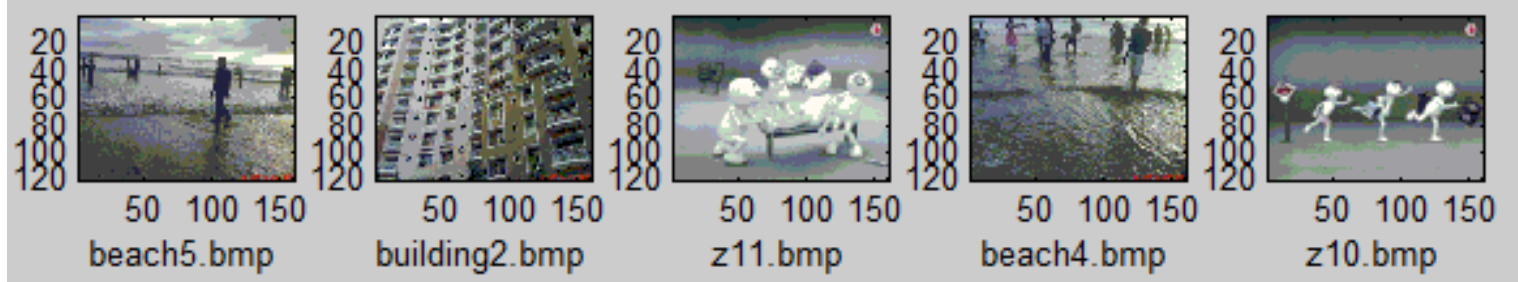

Figure 12. Sample output for texture search.
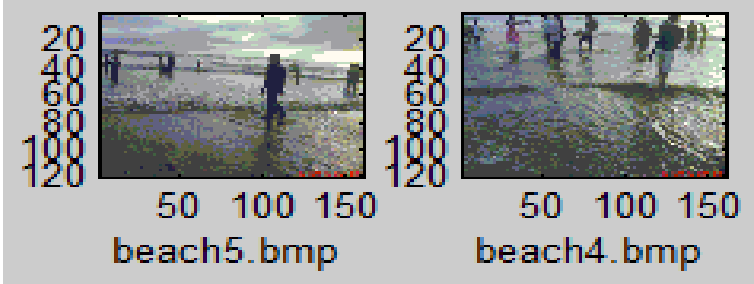

Figure 13. Sample output for entropy based search

\section{Content Based Satellite Cloud Image Retrieval: Application}

The satellite cloud image is a valuable source of information in weather forecasting and early prediction of different atmospheric disturbances such as typhoons, hurricanes etc. Due to the increased number and resolutions of the Earth imaging sensors and image acquisition techniques, the satellite image data is growing enormously which makes it difficult to store and manage. The traditional image retrieval technique is inefficient in retrieving these images. Content-based image retrieval is an approach from data mining community which provides the solution of managing this huge quantity of data. In this research, a ContentBased Image Retrieval (CBIR) system has been developed using gray level, texture and shape as retrieval features from the satellite image repository. The system allows the user to search for an image on the basis of any of the three features alone or in combination by assigning weights to the features. The histogram approach can be used to extract the gray level feature, texture feature is extracted using gray level co-occurrence matrix method and the shape feature is extracted using the morphological operations. The images and the extracted feature vectors can be stored in the Oracle $10 \mathrm{~g}$ database. Euclidean distance metric is used to compute the similarity between the images. The system is robust as it provides search based on the multiple features. The performance of the system can be evaluated by analyzing the retrieval results using precision. Content-Based Image Retrieval (CBIR) is based on the low level visual features [22] of the images these features are colour, texture and shape and spatial relation. Texture [23] and shape are important features in the meteorological satellite images. Different types of clouds have different shapes. The types of clouds associated with the Tropical Cyclone (TC) are the cumulonimbus types of clouds. Also the different objects in the meteorological satellite images such as typhoons, hurricanes can also be described by the shape [24] feature. There have been developed image retrieval systems for the remote sensing image archival. The focus of this research is to develop a CBIR system for the retrieval of the TC images from the meteorological satellite archival that can allow the meteorologists to study the past weather system and understand the current weather system. 


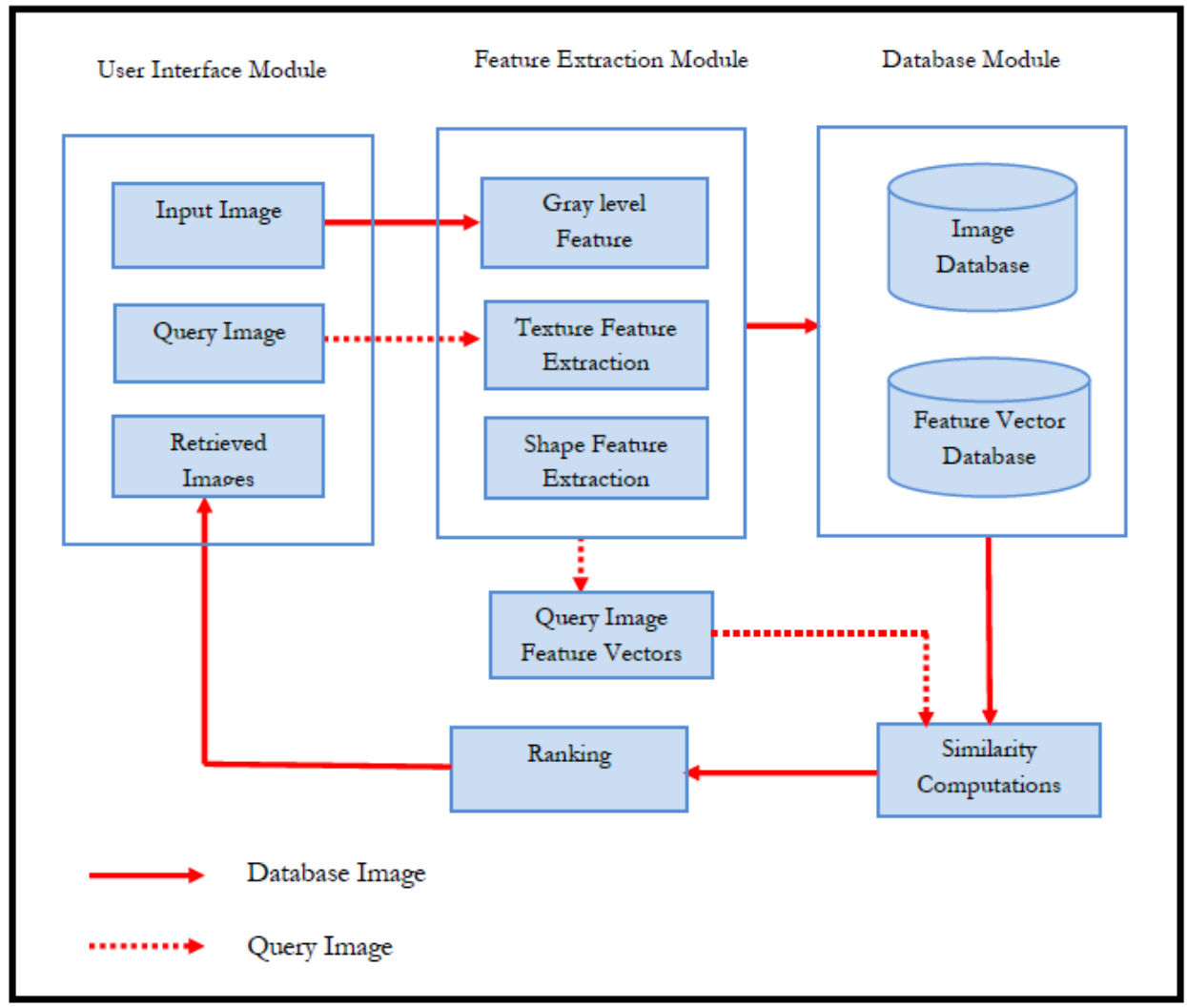

Figure 14. Workflow of methodology which can be adopted

\section{Conclusion And Future Work}

We have planned to incorporate the text based image retrieval into the current work for even more improving the retrieval efficiency. Since the CBIR system is based on the color feature, the retrieval results are directly and easy to tell the performances. In the future work, we hope to build a generalized query method which increase the system searching ability and provide more accurate content descriptions of places of interest places by performing color feature analysis and $\mathrm{CCH}$ image extraction simultaneously. As a result, the CBIR system will be able to suggest more relevant annotations and descriptions. Furthermore, we hope to optimize the system architecture and modules proposed in this paper. There exists some detail setting can be discussed and optimized with the images retrieval issues.

\section{Acknowledgements}

I would like to thank my guide and university for providing the consistent support for completion of this paper. Furthermore, I am also thankful to Director, HCTM, KAITHAL for making availability of resources and software for completion of this work.

\section{References}

[1]. N. S. Chang, and K. S. Fu, "A relational database system for images," Technical Report TR-EE 79-82, Purdue University, May 1979.

[2]. A.M.W. Smeulders, M. Worring, S. Santini, A.Gupta, and R. Jain, "Content-based image retrieval at the end of early years, "IEEE Trans. On Pattern Analysis and machine intelligence, vol. 22, no. 12, December 2000.

[3]. C. Carson, M. Thomas, S. Belongie, J. M. Hellerstein, and J. Malik, "Blobworld: A system for region-based image indexing and retrieval," In D. P. Huijsmans and A. W. M. Smeulders, ed. Visual Information and Information System, Proceedings of the Third International Conference VISUAL'99, Amsterdam, The Netherlands, June 1999, Lecture Notes in Computer Science 1614. Springer, 1999.

[4]. J. Assfalg, A. D. Bimbo, and P. Pala, "Using multiple examples for content-based retrieval," Proc. Int'l Conf. Multimedia and Expo, 2000.

[5]. S. K. Chang, C. W. Yan, D. C. Dimitroff, and T. Arndt, "An intelligent image database system," IEEE Trans. on Software Engineering, Vol.14, No.5, pp. 681-688, May 1988

[6]. S. K. Chang, Q. Y. Shi, and C. Y. Yan, "Iconic indexing by 2-D strings," IEEE Trans. on Pattern Anal. Machine Intell., Vol.9, No.3, pp. 413-428, May 1987.

[7]. C. Faloutsos et al, "Efficient and effective querying by image content," Journal of intelligent information systems, Vol.3, pp.231$262,1994$.

[8]. M. Flickner, H. Sawhney, W. Niblack, J. Ashley, Q. Huang, B. Dom, M. Gorkani, J. Hafner, D. Lee, D. Petkovic, D. Steele, and P. Yanker, "Query by image and video content: The QBIC system."IEEE Computer, Vol.28, No.9, pp. 23-32, Sept. 1995. 
[9]. N. S. Chang, and K. S. Fu, "Query by pictorial example," IEEE Trans. on Software Engineering, Vol.6, No.6, pp. 519-524, Nov.1980.

[10]. J. R. Bach, C. Fuller, A. Gupta, A. Hampapur, B. Horowitz, R. Humphrey, R. Jain, and C. F. Shu, "The virage image search engine: An open framework for image management," In Proc. SPIC Storage and Retrieval for Image and Video Database, Feb. 1996.

[11]. S. K. Chang, and T. L. Kunii, "Pictorial database systems," IEEE Computer Magazine, Vol. 14, No.11, pp.13-21, Nov.1981.

[12]. Kondekar V. H., Kolkure V. S., Kore S.N. "Image Retrieval Techniques based on Image Features: A State of Art approach for CBIR", International Journal of Computer Science and Information Security,Vol. 7, No. 1, 2010.

[13]. Ja-Hwung Su, Wei-Jyun Huang, Philip S. Yu, and Vincent S. Tseng, "Efficient Relevance Feedback for Content-Based Image Retrieval by Mining User Navigation Patterns" IEEE transactions on knowledge and data engineering, vol. 23, no. 3, march 2011.

[14]. Liana Stanescu, IADIS International Conference on Applied Computing, 2005 "on-line software system for content-based visual query of a color medical imagery."

[15]. K. Arbter, W. E. Snyder, H. Burkhardt, and G. Hirzinger, "Application of affine-invariant Fourier descriptors to recognition of 3D objects," IEEE Trans. Pattern Analysis and Machine Intelligence, vol. 12, pp. 640-647, 1990.

[16]. H. Burkhardt, and S. Siggelkow, "Invariant features for discriminating between equivalence classes," Nonlinear Model-based Image Video Processing and Analysis, John Wiley and Sons, 2000.

[17]. T. Chang, and C.C.J. Kuo, "Texture analysis and classification with tree-structured wavelet transform," IEEE Trans. on Image Processing, vol. 2, no. 4, pp. 429-441, October 1993.

[18]. I. Daubechies, "The wavelet transform, time-frequency localization and signal analysis," IEEE Trans. on Information Theory, Vol. 36, pp. 961-1005, Sept. 1990.

[19]. J. M. Francos, A. A. Meiri, and B. Porat, "A unified texture model based on a 2d Wold like decomposition," IEEE Trans on Signal Processing, pp.2665-2678, Aug. 1993.

[20]. Neetesh Gupta, R.K.Singh, “A New Approach for CBIR Feedback based Image Classifier", International Journal of Computer Applications (0975 - 8887) Volume 14- No.4, January 2011.

[21]. Rahul Metha, Nishkhol Mishra, Sanjeev Sharma, "Color - Texture based image retrieval system", International Journal of Computer Applications (0975 - 8887), Volume 24 - No. 5 June 2011.

[22]. Satya Sai Prakash, RMD. Sundaram. Combining Novel features for Content Based Image Retrieval. IEEE XPLORE DIGITAL LIBRARY pages: 373 - 376, 12 November 2007.

[23]. Wasim Khan, Shiv Kumar. Neetesh Gupta, Nilofar Khan, "A Proposed Method for Image Retrieval using Histogram values and Texture Descriptor Analysis", International Journal of Soft Computing and Engineering (IJSCE) ISSN: 2231-2307, Volume-I Issue-II, May 2011.

[24]. E. M. Arkin, L.P. Chew, D..P. Huttenlocher, K. Kedem, and J.S.B. Mitchell, "An efficiently computable metric for comparing polygonal shapes," IEEE Trans. Pattern Analysis and Machine Intelligence, vol. 13, no. 3, pp. 209-226, 1991. 